\title{
An Experimental Study on the Effect of Ambiguity in a Coordination Game*
}

\author{
David Kelsey \\ Department of Economics, \\ University of Exeter, England
}

\author{
Sara le Roux \\ Department of Economics, \\ Oxford Brookes University, England
}

27th December 2014

\begin{abstract}
We report an experimental test of the influence of ambiguity on behaviour in a coordination game. We study the behaviour of subjects in the presence of ambiguity and attempt to determine whether they prefer to choose an ambiguity safe option. We find that this strategy, which is not played in either Nash equilibrium or iterated dominance equilibrium, is indeed chosen quite frequently. This provides evidence that ambiguity aversion influences behaviour in games. While the behaviour of the Row Player is consistent with randomising between her strategies, the Column Player shows a marked preference for avoiding ambiguity and choosing his ambiguity-safe strategy.
\end{abstract}

Keywords: Ambiguity; Choquet expected utility; coordination game; Ellsberg urn, experimental economics.

JEL Classification: C72, C91, D03, D81

\footnotetext{
*We would like to thank Dieter Balkenborg, Adam Dominiak, Peter Dursch, Jürgen Eichberger, Todd Kaplan, Joshua Teitelbaum, Horst Zank, participants in seminars in Bristol, Exeter, Manchester and RUD Paris, and some anonymous referees for their comments and suggestions. We would like to thank Tim Miller for programming our Ellsberg Urn simulator.

$\dagger$ This paper is based on a chapter from Sara le Roux's PhD thesis.

${ }^{\ddagger}$ Address for Correspondence: Sara le Roux, Department of Accounting, Finance \& Economics, Oxford Brookes University, Wheatley Campus, Oxford OX33 1HX. E-mail: sle-roux@brookes.ac.uk
} 


\section{Introduction}

\section{$1.1 \quad$ Background}

This paper reports an experimental study of the impact of ambiguity in games. Decisions under uncertainty are said to be ambiguous if there are no objective probabilities given and it is difficult or impossible to assign subjective probabilities. There is by now a large experimental literature which shows that ambiguity makes a difference in single person decisions. For a survey see, Camerer and Weber (1992). However most economic decisions are not made by single individuals but by groups of individuals involved in strategic interactions. There is a small experimental literature on ambiguity in games. ${ }^{1}$ However, most of these papers do not test specific theories of the impact of ambiguity in games. It is thus difficult to predict what effect ambiguity has, and in which direction it will cause behaviour to change. Our research studies experimentally the comparative statics of ambiguity in games. In particular we test whether the theory of equilibrium under ambiguity (EUA, Eichberger, Kelsey, and Schipper (2009)) performs better than Nash equilibrium. ${ }^{2}$ We find that ambiguity does affect behaviour in games. Moreover ambiguity appears to have a larger impact in games than in single person decisions.

A game is a stylized way of representing a situation where a group of individuals have to make a number of linked decisions and thus forms a model of many economic interactions. Games provide a useful intermediate step between single person decisions and economic applications. Since many economic problems can be represented as games we believe this research will be useful for understanding the impact of ambiguity in economics.

\footnotetext{
${ }^{1}$ See for instance Colman and Pulford (2007), Eichberger, Kelsey, and Schipper (2008), Ivanov (2011), Kelsey and le Roux (2013) or Di Mauro and Castro (2011).

${ }^{2}$ The theory is based on earlier research by Dow and Werlang (1994) and Eichberger and Kelsey (2000).
} 


\subsection{The Experiments}

We consider a Battle of Sexes game which has an added safe strategy, $R$, available for Player 2, see below.

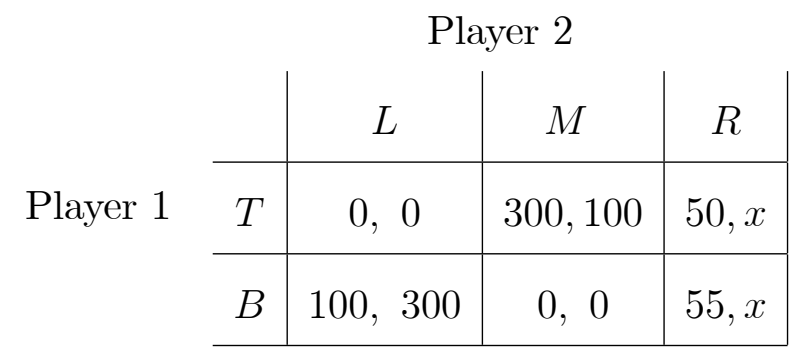

Table 1: Battle of the Sexes with a secure option

The value of $x$, which is the safe option available to Player 2, varies every round in the range $60-260$. For some values of $x$, the safe strategy (in our game, option $R$ ) is dominated by a mixed strategy of $L$ and $M$, and thus would not be played in a Nash equilibrium. For some higher values of $x$ the game is dominance solvable.

If players see a neutrally-framed Battle of Sexes game without a secure option, there are two pure Nash equilibria, neither of which is focal. Even if a player wishes to use a Nash equilibrium strategy they have to decide which one. Game theory does not provide guidance on how to choose. Thus we believe that it is possible that subjects will perceive considerable ambiguity in this game. Moreover, subjects played each round (with a varied $x$ ) just once, in our attempt to make the game one shot. No feedback was provided to subjects between rounds. We expected these conditions to lead subjects to perceive the games to be ambiguous.

The effect of ambiguity-aversion is to make $R$ (the ambiguity-safe option) attractive for Player 2. This is never chosen in Nash equilibrium for the parameter values considered by us. However it may be chosen when there is ambiguity. Moreover for some values of $x$, our games are dominance solvable and $R$ is not part of the equilibrium strategy. Despite this, we find that $R$ is chosen quite frequently by subjects. While the Row Player appears to be randomising 50 : 50 between her strategies, the Column Player shows a marked preference for avoiding ambiguity and choosing his ambiguity-safe strategy. Thus, ambiguity influences behaviour in the games. (We use the convention that female pronouns denote the row player and male 
pronouns denote the column player. $)^{3}$

\subsection{Results}

During the experiment, we alternated the Battle of Sexes games with decision problems based on the 3-ball Ellsberg urn. In these rounds, subjects were presented with an urn containing 90 balls, of which 30 were Red, and the remainder an unknown proportion of Blue or Yellow, and asked to pick a colour to bet on. The payoff attached to Red was varied in order to obtain an ambiguity threshold. Alternating experiments on urns and games had the dual aim of erasing the short term memory of subjects, and providing an independent measure of subjects' ambiguity-attitudes.

Subjects appeared to perceive a greater level of ambiguity in the two-person coordination game, than in the single person Ellsberg urn decision problem. We found that subjects chose the ambiguity-safe option $R$ in the game, but were unwilling to pay a penalty to choose $R e d$ (the unambiguous option in the urn experiment). There are a number of possible probability distributions which could describe the draw from the urn. Amongst these the one which assigns equal probability to each colour is a most natural focal point. In contrast in an experiment on games not only is the opponent's behaviour ambiguous, there is also no clear focal probability distribution.

Another difference is that in single person decision problems a proxy for ambiguity is introduced by the experimenter, using an artificial device such as the Ellsberg urn. However in games, ambiguity is created by the other subjects taking part in the experiment. Behaviour in the financial market is dependent on other people, and games can be used to effectively model such economic conditions. Natural disasters on the other hand, are more like single person decision problems.

If ambiguity-attitude is a fixed characteristic of the individual we would expect to see a positive correlation between choosing the ball with known probability in the urn experiment and choosing the safe strategy in games. In fact we found no statistically significant correlation,

\footnotetext{
${ }^{3}$ Of course this convention is for convenience only and bears no relation to the actual gender of subjects in our experiments.
} 
which suggests that ambiguity-attitude varies a lot from one context to another. The differences between the games and the urn experiments suggest that, even for a given subject, ambiguityattitude is quite variable depending on the type of decision (s)he is making. A consequence of this is that it will be difficult to test theories by measuring ambiguity-attitude in one context and then proceeding to use the measured ambiguity-attitude to predict behaviour in another context.

Organisation of the Paper In Section 2, we describe the theory being tested in the experiments. Section 3 describes the experimental design employed, Section 4 consists of data analysis and results, Section 5 reviews related literature and Section 6 provides a summary of results together with future avenues of research. The appendix contains the proofs of the main results.

\section{Preferences and Equilibrium under Ambiguity}

In this section we explain neo-additive preferences which we use to model ambiguity. The model is then developed into a theory of ambiguity in games.

\subsection{Modelling Ambiguity}

The Ellsberg paradox is a violation of the Subjective Expected Utility (SEU), Savage (1954). One version of the paradox is explained below. Consider an urn filled with 90 balls, 30 of which are red $(R)$ and the remaining 60 are of an unknown mix of blue $(B)$ and yellow $(Y)$. One ball is drawn at random, and the payoff depends on the colour of the ball drawn and the act chosen. Subjects are asked to choose between acts $f, g, f^{\prime}, g^{\prime}$ as shown in the table below (Pay-offs in Experimental Currency Units - ECU):

Subjects asked to choose between $f$ and $g$, generally prefer $f$ because of the definite $\frac{1}{3}$ chance of winning 100 ECU to the ambiguous act $g$, but when asked to choose between $f^{\prime}$ and 
Table 1: The Ellsberg Options

\begin{tabular}{|c|c|c|c|}
\hline \multirow{2}{*}{ Act } & 30 balls & \multicolumn{2}{|c|}{60 balls } \\
\cline { 2 - 4 } & Red $R$ & Blue $B$ & Yellow $Y$ \\
\hline$f$ & 100 & 0 & 0 \\
\hline$g$ & 0 & 100 & 0 \\
\hline$f^{\prime}$ & 100 & 0 & 100 \\
\hline$g^{\prime}$ & 0 & 100 & 100 \\
\hline
\end{tabular}

$g^{\prime}$, the same subjects prefer $g^{\prime}$, which gives a $\frac{2}{3}$ chance of winning 100 ECU, again avoiding the ambiguous act $f^{\prime}$.

These choices cannot be represented as maximising expected utility with respect to a standard subjective probability distribution $\pi$. However these preferences are compatible with nonadditive beliefs, introduced by Schmeidler (1989). Such beliefs may be represented by a capacity or non-additive set function $\nu$. In this case it is possible that $\nu(R \cup Y) \neq \nu(R)+\nu(Y)$, which could be compatible with the choices in the Ellsberg paradox. ${ }^{4}$ Schmeidler (1989) proposed a theory called Choquet Expected Utility (CEU), where outcomes are evaluated by a weighted sum of utilities, but unlike EUT the weights used depend on the acts. The model preserves additivity in beliefs when there is conventional risk, while permitting non-additivity for ambiguous events. Within CEU individuals can be either optimistic or pessimistic in their outlook towards ambiguity. An optimistic (resp. pessimistic) outlook would over-estimate the likelihood of a good (resp. bad) outcome - inducing one to make risky decisions such as investing in dotcom shares.

Neo-additive capacities were introduced by Chateauneuf, Eichberger, and Grant (2007). In this model the decision-maker has beliefs based on an additive probability distribution $\pi$. However (s)he lacks confidence in these beliefs hence they are ambiguous beliefs. The ambiguity is represented by the parameter $\delta$. The individual's attitude to ambiguity is represented by the parameter $\alpha$, with higher values of $\alpha$ corresponding to greater ambiguity-aversion. Consider a two-player game with a finite set of pure strategies $S_{i}$, such that $s_{i}$ is the player's own strategy and $S_{-i}$ denotes the set of possible strategy profiles for $i$ 's opponents. The payoff function of

\footnotetext{
${ }^{4}$ If $v(R)>v(B)$ and $v(R \cup Y)<v(B \cup Y)$, then these beliefs will be compatible with the Ellsberg Paradox.
} 
player $i$ is denoted $u_{i}\left(s_{i}, s_{-i}\right)$. The functional form of preferences may be represented as:

$$
V_{i}\left(s_{i} ; \pi_{i}, \alpha_{i}, \delta_{i}\right)=\delta_{i}\left(1-\alpha_{i}\right) M_{i}\left(s_{i}\right)+\delta_{i} \alpha_{i} m_{i}\left(s_{i}\right)+\left(1-\delta_{i}\right) \int u_{i}\left(s_{i}, s_{-i}\right) d \pi_{i}\left(s_{-i}\right)
$$

where $M_{i}\left(s_{i}\right)=\max _{s_{-i} \in S_{-i}} u_{i}\left(s_{i}, s_{-i}\right)$ and $m_{i}\left(s_{i}\right)=\min _{s_{-i} \in S_{-i}} u_{i}\left(s_{i}, s_{-i}\right){ }^{5}$ These preferences maximise a weighted average of the best payoff, the worst payoff and the expected payoff. They are a special case of CEU. They are also a special case of the $\alpha$-MEU model, ?, which represents ambiguity by a set of probability distributions and ambiguity-attitude by the parameter $\alpha$ expressing the weight given to the minimum possible expected utility.

Intuitively, $\pi$ can be thought to be the decision-maker's belief. However, he is not sure of this belief, hence it is an ambiguous belief. His confidence about it is modelled by $\left(1-\delta_{i}\right)$, with $\delta_{i}=1$ denoting complete ignorance and $\delta_{i}=0$ denoting no ambiguity. His attitude to ambiguity is measured by $\alpha_{i}$, with $\alpha_{i}=1$ denoting pure pessimism and $\alpha_{i}=0$ denoting pure optimism. If the decision-maker has $0<\alpha_{i}<1$, he is neither purely optimistic nor purely pessimistic (i.e., ambiguity-averse), but reacts to ambiguity in a partly pessimistic way by putting a greater weight on bad outcomes and in a partly optimistic way by putting a greater weight on good outcomes.

\subsection{Equilibrium under Ambiguity}

In this section we present an equilibrium concept for strategic games with ambiguity. In a Nash equilibrium, players are believed to behave in a manner that is consistent with the actual behaviour of their opponents. They perfectly anticipate the actions of their opponent and can thus provide a best response to it in the form of their own action. However, for non-additive beliefs, the Nash idea of having consistent beliefs regarding the opponent's action and thus being able to play a best response to these beliefs, needs to be modified. We assume that players choose pure strategies. In equilibrium, a player's beliefs about the pure strategies of his/her opponent must be best responses for that opponent, given the opponent's beliefs.

\footnotetext{
${ }^{5}$ Note that Chateauneuf, Eichberger, and Grant (2007) write a neo additive capacity in the form $\mu(E)=$ $\delta \alpha+(1-\delta) \pi(E)$. We have modified their definition to be consistent with the majority of the literature where $\alpha$ is the weight on the minimum expected utility.
} 
Unlike Nash equilibrium where a player can assign an additive probability distribution to his/her opponent's actions, ambiguous beliefs are represented by capacities. The support of a capacity is a player's belief of how the opponent will act. Formally, the support of a neo-additive capacity, $\nu(A)=\delta \alpha+(1-\delta) \pi(A)$, is defined by $\operatorname{supp}(\nu)=\operatorname{supp}(\pi)$. Thus the support of a neo-additive belief is equal to the support of its additive component. ${ }^{6}$

Definition 2.1 (Equilibrium under Ambiguity) A pair of neo-additive capacities $\left(\nu_{1}^{*}, \nu_{2}^{*}\right)$ is an Equilibrium Under Ambiguity (EUA), for $i=1,2$ and $\operatorname{supp}\left(\nu_{i}^{*}\right) \subseteq R_{-i}\left(\nu_{-i}^{*}\right)$, where $R_{i}$ denotes the best-response correspondence of player $i$, given that his/her beliefs are represented by $\nu_{i}$ and is defined by:

$$
R_{i}\left(\nu_{i}\right)=R_{i}\left(\pi_{i}, \alpha_{i}, \delta_{i}\right):=\operatorname{argmax}_{s_{i} \in S_{i}} V_{i}\left(s_{i} ; \pi_{i}, \alpha_{i}, \delta_{i}\right)
$$

This definition of equilibrium is taken from Eichberger, Kelsey, and Schipper (2009), who adapt an earlier definition in Dow and Werlang (1994). These papers show that an EUA will exist for any given ambiguity-attitudes for the players. In games, one can determine $\pi_{i}$ endogenously as the prediction of the players from the knowledge of the game structure and the preferences of others. In contrast, we treat the degrees of optimism, $\alpha_{i}$ and ambiguity, $\delta_{i}$, as exogenous. In equilibrium, each player assigns strictly positive likelihood to his/her opponent's best responses given the opponent's belief. However, each player lacks confidence in his/her likelihood assessment and responds in an optimistic way by over-weighting the best outcome, or in a pessimistic way by over-weighting the worst outcome.

Alternative approaches to equilibrium with ambiguity can be found in Klibanoff (1993) and Lo (1996). They model players as having preferences which satisfy the axioms of maxmin expected utility (MMEU, Gilboa and Schmeidler (1989)). Players are allowed to have beliefs which are represented by sets of conventional probability distributions. As such, players can have mixed strategies that are chosen from these sets of probabilities. They model ambiguity aversion as a strict preference among players to randomise between strategies when they are

\footnotetext{
${ }^{6}$ This definition is justified in Eichberger and Kelsey (2014).
} 
indifferent between two pure strategies.

\section{Experimental Model}

\subsection{Battle of the Sexes Game}

In this section, we explain the games used in our experimental sessions. These are similar to the standard battle of the sexes game, except that they have been modified by giving the column player an additional option which is secure.

As we argue in the introduction, we believe ambiguity will be high due to the multiplicity of equilibria in the basic Battle of the Sexes game. When $x=60, R$ is dominated by a mixed strategy and hence is not played in Nash equilibrium or iterated dominance equilibrium.

The following results finds the Nash equilibria in our games. This sets a benchmark from which we can measure the effect of ambiguity.

Proposition 3.1 Our modified battle of sexes game has the following Nash equilibria

1. When $0 \leqslant x \leqslant 75$, there are 3 equilibria: $(T, M),(B, L)$ and a mixed strategy equilibrium $\left(\frac{3}{4} \cdot T+\frac{1}{4} \cdot B, \frac{1}{4} \cdot L+\frac{3}{4} \cdot M\right) ;^{7}$

2. When $75<x \leqslant 100$, there are 3 equilibria: $(T, M),(B, L)$ and $\left(\frac{x}{100} \cdot T+\frac{100-x}{100} \cdot B, \frac{1}{61}\right.$. $\left.M+\frac{60}{61} \cdot R\right)$

3. When $100<x<300$, there is a Nash unique equilibrium: $(B, L)$, which is also an iterated dominance equilibrium.

Ambiguity about the behaviour of Player 1 would make the secure option $R$ more attractive for Player 2. Note that the best response to $R$, is for Player 1 to play $B$. Hence of the two possible Nash equilibria $(T, M)$ and $(B, L)$, the latter may be more robust to ambiguity. ${ }^{8}$

\footnotetext{
${ }^{7}$ The notation $\frac{3}{4} \cdot T+\frac{1}{4} \cdot B$ denotes the mixed strategy where $T$ is played with probability $\frac{3}{4}$ and $B$ is played with probability $\frac{1}{4}$.

${ }^{8}$ Proposition 2 confirms that $(B, L)$ is an equilibrium for a greater parameter range than $(T, M)$.
} 
As described in Section 2.1, in CEU, convex capacities are used to model a pessimistic outlook to ambiguity. Neo-additive capacities are a special case of CEU, where the functional form of preferences may be represented as:

$$
V_{i}\left(s_{i} ; \pi_{i}, \alpha_{i}, \delta_{i}\right)=\delta_{i}\left(1-\alpha_{i}\right) M_{i}\left(s_{i}\right)+\delta_{i} \alpha_{i} m_{i}\left(s_{i}\right)+\left(1-\delta_{i}\right) \int u_{i}\left(s_{i}, s_{-i}\right) d \pi_{i}\left(s_{-i}\right)
$$

where $M_{i}\left(s_{i}\right)=\max _{s_{-i} \in S_{-i}} u_{i}\left(s_{i}, s_{-i}\right)$ and $m_{i}\left(s_{i}\right)=\min _{s_{-i} \in S_{-i}} u_{i}\left(s_{i}, s_{-i}\right)$. These preferences maximise a weighted average of the best payoff, the worst payoff and the expected payoff. Intuitively, $\pi$ is the decision-maker's ambiguous belief. His confidence about the ambiguous belief is modelled by $\left(1-\delta_{i}\right)$, with $\delta_{i}=1$ denoting complete ignorance and $\delta_{i}=0$ denoting no ambiguity. His attitude to ambiguity is measured by $\alpha_{i}$, with $\alpha_{i}=1$ denoting pure pessimism and $\alpha_{i}=0$ denoting pure optimism. If the decision-maker has $0<\alpha_{i}<1$, he is neither purely optimistic nor purely pessimistic (i.e., ambiguity-averse). We assume that the beliefs of the players may be represented by neo-additive capacities and that players are ambiguity-averse, i.e., $\alpha=1$.

Proposition 3.2 The game has the following Equilibria under Ambiguity for all $\delta_{i}$, where $\delta_{i}$, is the degree of ambiguity of player $i$ :

1. when $0 \leqslant x \leqslant\left(1-\delta_{i}\right) 75$, there are 3 equilibria, $(T, M),(B, L)$ and $\left(\frac{3}{4} \circ T+\frac{1}{4} \circ B, \frac{1}{4} \circ L+\right.$ $\left.\frac{3}{4} \circ M\right)$

2. when $\left(1-\delta_{i}\right) 75<x \leqslant\left(1-\delta_{i}\right) 100$, there are 3 equilibria: $(T, M),(B, L)$ and

$$
\left(\frac{x}{\left(1-\tilde{\delta}_{2}\right) 100} \circ T+\frac{\left(\left(1-\tilde{\delta}_{2}\right) 100\right)-x}{\left(1-\tilde{\delta}_{2}\right) 100} \circ B, \frac{1}{61} \circ M+\frac{60}{61} \circ R\right) ;
$$

3. when $\left(1-\delta_{i}\right) 100<x<\left(1-\delta_{i}\right) 300$, there is a unique equilibrium: $(B, L)$;

4. when $x>\left(1-\delta_{i}\right) 300$, there is a unique equilibrium: $(B, R)$.

Suppose $\delta_{i}=0.5$, which is compatible with the findings of Kilka and Weber (2001). Then (a) occurs for $0 \leqslant x \leqslant 37.5,(b)$ occurs for $37.5 \leqslant x \leqslant 50,(c)$ occurs for $50 \leqslant x \leqslant 150$ and $(d)$ occurs for $150 \leqslant x$. 
The testable hypothesis that arises from the analysis, is that while Nash equilibrium predicts that $R$ cannot be chosen in the range $37.5<x<50$ or $150<x<300$, EUA predicts $R$ can be chosen in these ranges.

\subsection{Ellsberg Urn Experiments}

The Battle of Sexes game was alternated with single person decision problems similar to the Ellsberg Paradox. Subjects were presented with an urn containing 90 balls, of which 30 were Red, and the remainder an unknown proportion of Blue or Yellow. Subjects were asked to pick a colour, and a ball was drawn from the urn. If the colour of the ball matched the colour chosen by the subject, it entitled the subject to a prize. The decisions put to the subjects took the following form:

"An urn contains 90 balls, of which 30 are Red. The remainder are either Blue or Yellow.

Which of the following options do you prefer?

a) Payoff of $y$ if a Red ball is drawn.

b) Payoff of 100 if a Blue ball is drawn.

c) Payoff of 100 if a Yellow ball is drawn."

Payoff " $y$ " attached to the option Red was changed from round to round, with $y=95,90$ or 80 , to measure the ambiguity threshold of subjects. In addition, we also put before subjects the classic case of Ellsberg Paradox, when $y=100$, as described in Table 3.

\section{Experimental Design}

The Battle of Sexes game and Ellsberg Urn problem described above were used in two series of paper-based experiments, one conducted at St. Stephen's College in New Delhi, India, and the other at the Finance and Economics Experimental Laboratory in Exeter (FEELE), UK. We conducted two sets of experiments in order to check that the response to ambiguity was consistent across locations.

Sessions 1 and 2 consisted of 20 subjects each. Sessions 3 and 4 consisted of 18 and 22 
subjects respectively. In total there were 80 subjects who took part in the experiment, 38 of which were females and 42 were males. We were also interested in whether or not participants had a mathematical background - of those taking part in the sessions, 45 studied a quantitative subject such as Biochemistry, Electronic Engineering or Astrophysics, while 35 studied a nonquantitative subject such as History, Philosophy, or International Relations. Each session lasted a maximum of 45 minutes.

Subjects were allowed to read through a short but comprehensive set of instructions at their own pace ${ }^{9}$, following which the instructions were also read out to all the participants in general. The subjects were then asked to fill out some practice questions to test their understanding of the games, before the actual set of experimental questions were handed out. At the start of the experiment, subjects were randomly assigned the role of either a Row Player or a Column Player for the purpose of the Battle of Sexes game, and remained in the same role throughout the rest of the experiment.

The experiment consisted of 11 rounds, starting with a decision regarding a Battle of Sexes game, which was then alternated with an Ellsberg Urn decision, such that there were in total 6 Battle of Sexes rounds and 5 Ellsberg urn decisions to be made. Each subject had to choose one option per round: Top/Bottom if they were a Row Player or Left/Middle/Right if they were a Column Player, and in case of the Ellsberg urn rounds Red, Blue or Yellow.

The values of $x$, the ambiguity-safe payoff available to the Column Player that were used for the Battle of Sexes game rounds were: 230,120, 200, 170, 260, 60 (in that order). In the first three Ellsberg urn rounds, the pay-offs attached to drawing a Blue or Yellow ball were held constant at 100, while those attached to drawing a Red ball varied as 95, 90, 80. The last two Ellsberg urn rounds consisted of the classic case of the Ellsberg paradox, where subjects had to choose between a payoff of 100 for a Red or 100 for a Blue ball, followed by a choice between a payoff of 100 for drawing a Red/Yellow ball or 100 for drawing a Blue/Yellow ball.

Once subjects had made all 11 decisions, a throw of dice determined one Battle of Sexes round and one Ellsberg urn round for which payments were to be made. Row Players' decisions

\footnotetext{
${ }^{9}$ Experimental protocols can be found here: http://saraleroux.weebly.com/experimental-protocols.html
} 
were matched against the Column Players' decisions according to a random and anonymous matching, and pay-offs were announced..$^{10}$

Rather than using a real urn we simulated the draw from the urn on a computer. ${ }^{11}$ The computer randomly assigned the number of blue and yellow balls in the urn so that they summed to 60 , while keeping the number of red balls fixed at 30 and the total number of balls in the urn at $90 .{ }^{12}$ It then simulated an independent ball draw for up to 30 subjects. If the colour of the ball drawn by the computer matched that chosen by the subject, it entitled him to the payoff specified in the round chosen for payment.

The total earnings of a subject was the sum of a show-up fee, payoff earned in the chosen Battle of Sexes round and payoff earned in the chosen Ellsberg urn round. Average payment made to Indian subjects was $R s .420$ ( 66 approximately), and to Exeter subjects was $£ 7.40$.

\section{Data Analysis and Results}

The levels of coordination in the Battle of Sexes game without the secure option, was about $50 \%$ (See Table 2), which is reasonable in an experimental game. To measure coordination we discard those occasions on which Player 2 chose the secure option. We measure coordination as the ratio of sucessful coordinations, i.e. either $\langle T . M\rangle$ or $\langle B, L\rangle$ to the total number of plays $\langle T . M\rangle$ and $\langle B, L\rangle,\langle T, L\rangle$ and $\langle B, M\rangle$, i.e.

$$
\frac{\operatorname{prob}(T, M)+\operatorname{pob}(B, L)}{\operatorname{prob}(T, M)+\operatorname{prob}(T, L)+\operatorname{prob}(B, L)+\operatorname{prob}(B, M)} .
$$

Sara Since these are observations it is misleading to describe them as probabilities. They are rather proportions of the actual data.

The players managed to coordinate on the pure Nash equilibria, $(T . M)$ and $(B, L)$, about

\footnotetext{
${ }^{10}$ For the purpose of the Indian experiments, $1 E C U=R s .1$, while for the Exeter experiments, $100 E C U=£ 2$. In addition, a show-up fee of $R s .250$ was paid to the Indian subjects and $£ 5$ to the Exeter subjects.

${ }^{11}$ The computer simulated urn can be found at the following link: http://saraleroux.weebly.com/experimentalprotocols.html

${ }^{12}$ The programme was produced by the FEELE lab programmer, Tim Miller. Even the experimenters were unaware of the processes determining the composition of the urn.
} 
$50 \%$ of the time, which appears normal for this type of experiment.

Table 2: Coordination in the Battle of Sexes Game

\begin{tabular}{|c|c|c|c|c|c|}
\hline$x=60$ & $x=120$ & $x=170$ & $x=200$ & $x=230$ & $x=260$ \\
\hline $50 \%$ & $50 \%$ & $51 \%$ & $41 \%$ & $60 \%$ & $43 \%$ \\
\hline
\end{tabular}

\subsection{Behaviour of the Row Player}

In the Battle of Sexes rounds of the experiment, the task of the Row Player was to choose between $T$ and $B$. We find that they individually and on aggregate, randomise closely to 50 : 50. See Figure 1, for a summary of the Row Player's behaviour.

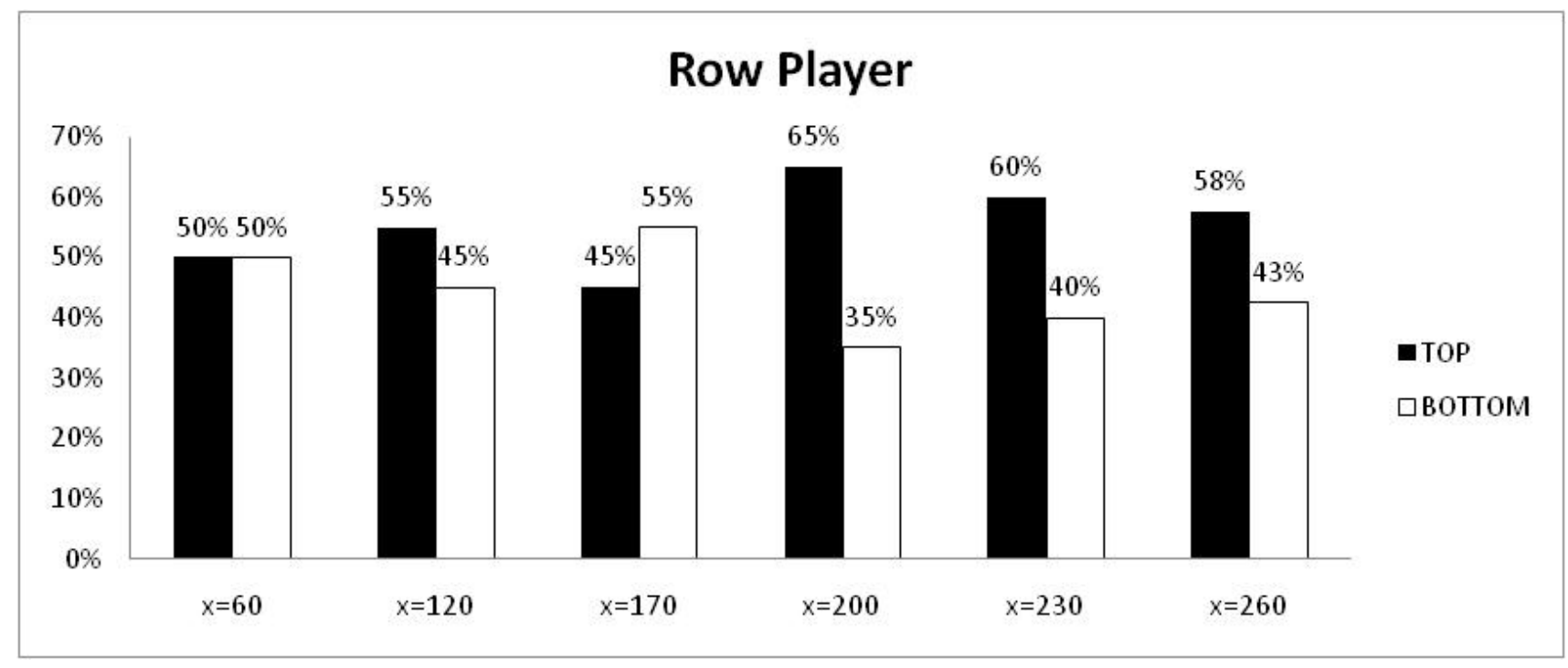

Figure 1: Row Player Behaviour

We conducted a binomial test with the null that the Row Player randomises 50 : 50 between $T$ and $B$, for each value of $x$. We fail to reject this hypothesis for each individual session even at a $10 \%$ level of significance. When tested for each value of $x$ on the whole (as a sum of all sessions combined), we fail to reject the null for all the values of $x$, except when $x=200$, where we reject the null at $5 \%$. In the case where $x=200$, the Row Player plays $T$ significantly more often than $B$. This is puzzling, since $B$ would be the best response to the Column Player choosing $R$. A possible reason why the Row Player might choose $T$, might be that the total payoff from $T$ is greater than that of playing $B$. 
We conducted a chi-squared test with the null hypothesis that the Row Player chooses $T$ and $B$ with equal probability $\left(H_{0}: \operatorname{prob}(T)=0.5, \operatorname{prob}(B)=0.5\right)$ versus the alternative that this is not true $\left(H_{1}: \operatorname{prob}(T) \neq 0.5\right)$. Again, we fail to reject this hypothesis even at the $10 \%$ level.

\subsection{Behaviour of the Column Player}

In the Battle of Sexes rounds of the experiment, the task of the Column Player was to choose between $L, M$ and the ambiguity-safe option $R$. See Figure ??, for a summary of the Column Player's behaviour.

When $x=60$ one might expect the Column Player to pick $L$, since $L$ has a much higher maximum pay-off than $M$. As seen in Figure ?? most subjects do indeed choose $L$. However, even at this low value of $x$, where the ambiguity-safe option $R$ is dominated by randomisation between the other strategies, a significant $30 \%$ of subjects still choose it. ${ }^{13}$ What is more interesting to note however, is that the number of subjects playing $R$, steadily increases from $73 \%$ to $98 \%$ for $120 \leqslant x \leqslant 260$. Nash equilibrium predicts that $R$ cannot be chosen for any of these values, but it is the clear choice of a majority of subjects in the presence of ambiguity, as seen in Figure ??.

According to Proposition 2, when $x=120$, neither EUA nor Nash predicts that $R$ would be chosen, if $\delta_{i}=0.5$ (as estimated by Kilka and Weber (2001)). In our data, we notice a large number of subjects $(73 \%)$ select $R$ when $x=120$. This would be compatible with EUA if $\delta_{i}=0.6$ (i.e., for a slightly higher $\delta_{i}$ than estimated by the Kilka and Weber (2001) study). If $\delta_{i}=0.6$, then for Proposition $2,(a)$ occurs for $0 \leqslant x \leqslant 30,(b)$ occurs for $30 \leqslant x \leqslant 40,(c)$ occurs for $40 \leqslant x \leqslant 120$ and $(d)$ occurs for $120 \leqslant x$. Nash equilibrium would predict that $R$ cannot be chosen in the range $120<x<300$, while EUA would predict that $R$ can be chosen for $x \geqslant 120$. Thus, our data captures the preferences of some individuals who had a slightly

\footnotetext{
${ }^{13}$ Subjects remained in the same role throughout the game. It is thus possible that subjects could consider the properties of the game more fully, as the rounds progressed, even though they were not given any feedback. It may be noted that this "repeated" nature of play may have led to more Column Players choosing the option $R$ when $x=60$, than is expected, as it was the last game round. These subjects may have got used to choosing $R$ and not re-optimised for the lower value of $x$.
} 
higher $\delta$ parameter, than those who took part in the Kilka and Weber (2001) study, and is compatible with EUA for $\delta_{i}=0.6$.

We conducted a binomial test with the null that the Column Player chooses $R$ as often as he does $L+M\left(H_{0}: \operatorname{prob}(\right.$ Right $)=0.5, \operatorname{prob}($ Left + Middle $\left.)=0.5\right)$, against the alternative that he plays $R$ more often than both $L+M$ combined $\left(H_{1}: \operatorname{prob}(\operatorname{Right})>\operatorname{prob}(L e f t+M i d d l e)\right)$, for each value of $x \cdot{ }^{14}$ We reject the null at a $1 \%$ level of significance for all the values of $x$ in the range $120-260$. This leads us to conclude that subjects play $R$ significantly more often than both $L$ and $M$ combined, at a $1 \%$ level of significance.

A chi-squared test with the null hypothesis that the Column Player chooses $R$ and $L+$ $M$ with equal probability $\left(H_{0}: \operatorname{prob}(\right.$ Right $)=0.5, \operatorname{prob}($ Left + Middle $\left.)=0.5\right)$ versus the alternative that this is not true $\left(H_{1}: \operatorname{prob}(\operatorname{Right}) \neq 0.5\right)$ is also rejected at the $1 \%$ level of significance, since $R$ is chosen significantly more often.

We ran a probit regression to ascertain what factors influenced subjects in choosing $R$ more often than $L$ or $M$. Dummy variables were defined to capture the characteristics of the data such as: $M a t h=1$, if the subject was doing a quantitative degree (Math $=0$, for degrees like English, History, Philosophy, Politics etc.); Male = 1, if gender is male (0, otherwise); Delhi $=1$, if the session was run in India (0, for Exeter); $x_{-} 120, x_{-} 170, x_{\_} 200, x_{\_} 230$, $x \_260=1$, depending on the value " $x$ " took in that particular round. ${ }^{15}$

A probit regression of Right on Math, Male, and the various $x$ - value dummies $x_{-}$120, $x \_170, x \_200, x \_230, x \_260$, has a chi-square ratio of 75.55 with a p-value of 0.0001 , which

\footnotetext{
${ }^{14}$ The binomial test was conducted for each value of $x$ except $x=60$, where EUA predicts that the column player can play $L$. It may be noted that for $x=60$, subjects play $L+M$ more than $50 \%$ of the time.

${ }^{15}$ The dummy for $x_{-} 60$ was dropped from the probit regression, in order to avoid the dummy variable trap.
} 
shows that our model as a whole is statistically significant. ${ }^{16}$ Regression results are seen below. ${ }^{17}$

$$
\begin{aligned}
\text { Right }= & -0.6236+0.538 \text { Math }-0.402 \text { Male }+1.16\left(x \_120\right)+1.08\left(x_{-}{ }^{170}\right) \\
& +1.75\left(x \_200\right)+2.27\left(x \_230\right)+2.57\left(x \_260\right)
\end{aligned}
$$

All the variables in the probit regression were individually statistically significant. We note that if a subject had a quantitative degree, (s)he is more likely to choose $R$. Moreover, males are less likely to opt for $R$ than females, and as the value of $x$ increases, the subject is more likely to pick the ambiguity-safe option.

Next we consider alternative explanations for our results. A leading possibility is the cognitive hierarchy model (Camerer, Ho, and Chong (2004)). Consider the case where $x=200$. At this value of $x$, a majority of Column Players choose to play $R$, even though $R$ is not chosen in a Nash equilibria. We now consider a cognitive-heirarchy, where Level - 0 Column Players play $R$ with probability $\frac{1}{3}$, and Level - 0 Row Players choose $T$ and $B$ with probability $50: 50$. Level - 1 Column Players would then choose to play $R$ with probability 1 , and Level - 1 Row Players would play $B$ with probability 1 . If Level -2 Column Players best respond to a belief that Row Players are some mix of Level -0 and Level - 1, it would lead them to choose $R$, if they think the chance of Row being Level - 1 is high enough. This argument suggests that a Cognitive Hierarchy Model (CHM) where most Column Players are Level - 1 or 2, could also explain the frequent play of $R$ in our game.

The disadvantage of using CHM is that it allows players to have arbitrary levels of rationality, which can be adjusted freely. As such, it is unsurprising that it can be better fitted to data. The neo-additive preferences we use in EUA are derived from an axiomatic decision theory.

\footnotetext{
${ }^{16}$ An initial probit regression, showed that the dummy variable for location (Delhi/Exeter) was not significant. This re-inforced our beliefs that the behaviour of the Indian subjects was very similar to that of the Exeter subjects. Thus, the location dummy variable was dropped and the model was re-run without it.

${ }^{17}$ The coefficients from a probit regression do not have the same interpretation as coefficients from an Ordinary Least Squares regression. From the probit results, we can interpret that if a subject had a quantitative degree, his z-score increases by 0.538 , making him more likely to pick $R$. If the subject is male, the z-score decreases by 0.402 , hence males are less likely to opt for the ambiguity-safe option $R$ than females. When $x=120$ : the z-score increases by 1.16 , for $x=170$ : the $\mathrm{z}$-score increases by 1.08 , for $x=200$ : the z-score increases by 1.75 , for $x=230$ : the $\mathrm{z}$-score increases by 2.27 , for $x=260$ : the z-score increases by 2.57 ; more than the base which is $x=60$.
} 
There is no corresponding axiomatic theory behind level- $k$ equilibrium. In this respect, EUA is more rigorous, in that it puts constraints on the parameters measuring the ambiguity attitude and the level of ambiguity faced by subjects. Moreover as Kilka and Weber (2001) show these parameters can be measured. A person's ambiguity attitude ( $\alpha$-parameter, capturing optimism/pessimism) is a personal characteristic, and may be taken as given. The level of ambiguity ( $\delta$-parameter) on the other hand, is dependant on the situation he/she is facing. For instance, the level of ambiguity experienced in a one-shot game would be much higher than that of a repeated game, where players can update beliefs to incorporate learning. Hence, the testable hypothesis generated by EUA does not have an analogous counter part for the CHM model. In particular, the advantage of using EUA is that it can explain diverging behaviour in a number of games, using the same set of ambiguity and ambiguity-attitude parameters. See Eichberger, Kelsey, and Schipper (2008) or Kelsey and le Roux (2013) for experimental tests of EUA on other games.

\subsection{Player Behaviour in the Ellsberg Urn Rounds}

The Ellsberg Urn rounds were alternated with the Battles of Sexes rounds. This was designed to test whether there was a correlation between ambiguity-averse behaviour in the game and ambiguity attitude in single person decision problems. ${ }^{18}$

As can be seen in Figure ??, subjects chose Blue and Yellow coloured balls (the ambiguous option) more often than they chose Red. ${ }^{19}$ This was designed to test whether subjects who chose Right (the ambiguity-safe option) in the Battle of Sexes rounds, would choose Red (the colour with the unambiguous number of balls) in the Urn rounds. In practice, the observed correlation was weak. ${ }^{20}$

One notable feature of this data is the low level of ambiguity-aversion compared to previous studies. In the case where $y=100$ our results are comparable to the previous literature. For

\footnotetext{
${ }^{18}$ We would like to thank Peter Dursch, whose suggestions helped the design of the experiment.

${ }^{19}$ The data for $y=100$ is from the classic Ellsberg paradox round. It is not completely comparable, as subjects were not given the option of choosing yellow.

${ }^{20}$ Only 12 of the 80 subjects that took part in our experiment always chose Red, 3 of these subjects were Row Players and thus do not have an ambiguity-safe option. As such, the Pearson correlation between choosing Right in the game round and Red in the Urn round was close to zero.
} 
lower values of $y$, subjects have to pay a monetary penalty to avoid ambiguity. Even small penalties produced a large drop in the number of subjects choosing the unambiguous option. Of the 57 subjects who chose Red when $y=100,29$ switched away from Red when $y=95$, 7 switched at $y=90,6$ switched at $y=80$, while 12 subjects never switched away from Red (even at lower levels of $y$ ). ${ }^{21}$ Moreover for values of $y=95 / 90 / 80$, of the 80 subjects that took part in the experiment, only 12 always chose Red, 11 chose Red twice, 20 chose Red once, and a significant 37 subjects never chose Red - always opting for either Blue or Yellow, the ambiguous options.

It is interesting to note that even in the round where the payoff attached to Red was 80 $E C U$, a large minority (30\%) still chose the unambiguous option, despite facing a substantial monetary penalty. Of the 12 subjects who always picked Red, 3 are Row Players and so not relevant to our discussion. The remaining 9 are Column Players: 7 of these always chose the ambiguity-safe combination of Right-Red (not considering their choice when $x=60$ ), while 2 chose Left/Middle/Right while always picking Red.

We conducted a binomial test with the null that Red was chosen as often as Blue+Yellow combined $\left(H_{0}: \operatorname{prob}(R e d)=0.5, \operatorname{prob}(\right.$ Blue + Yellow $\left.)=0.5\right)$, against the alternative that Blue + Yellow was chosen more often $\left(H_{1}: \operatorname{prob}(B l u e+Y e l l o w)>\operatorname{prob}(R e d)\right)$. We reject the null at a $5 \%$ level of significance when the payoff attached to Red $=95$, and at $1 \%$ level of significance when Red $=90 \& 80$. Looking at subject choices on the whole, over the three rounds, we can reject the null at a $1 \%$ level of significance.

Thus, the ambiguous options Blue and Yellow are chosen significantly more often than Red, which leads us to speculate whether the penalty for choosing Red was set too high or whether subjects are mildly ambiguity-seeking in the Ellsberg urn rounds, even though they appear to be ambiguity-averse in the Battle of Sexes rounds. A probit regression run to investigate whether gender, location or degree subject affected subjects' choice of Blue and Yellow was inconclusive and none of these potential explanatory variables were found to be significant.

\footnotetext{
${ }^{21}$ We ignore the subjects who switch multiple times between Red and Blue/Yellow. Such subjects may have been (incorrectly) using the notion of diversifying a portfolio.
} 


\subsection{Classic Ellsberg Paradox Rounds}

In the last two urn rounds, the classic Ellsberg Paradox was put before the subjects. As can be seen from Table 3, a majority of them preferred Red to Blue, followed by the choice Blue/Yellow (rather than Red/Yellow). 38 of the 80 (48\%) subjects chose Red followed by Blue/ Yellow, thus displaying the Classic Ellsberg Paradox. The opposite preference was expressed by 7 (9\%) of the subjects. They subjects chose Blue followed by Red/Yellow, which indicates an ambiguity preference. Looking strictly at the Column Players who display the Ellsberg Paradox ${ }^{22}$ : 16 (67\%) subjects always chose the ambiguity-safe option Right - but these people do choose Blue/Yellow when the payoff attached to Red $=95,90,80$, while 8 (33\%) play a mixture of Left/Middle/Right.

Table 3: Player Behaviour in Classic Ellsberg Paradox Rounds

\begin{tabular}{|c|c|c|}
\hline Choice & \multicolumn{2}{|c|}{ Response } \\
\hline Red followed by Red/Yellow & 19 & $24 \%$ \\
\hline Red followed by Blue/Yellow & 38 & $48 \%$ \\
\hline Blue followed by Blue/Yellow & 16 & $20 \%$ \\
\hline Blue followed by Red/Yellow & 7 & $9 \%$ \\
\hline
\end{tabular}

\section{Related Literature}

In this section we review the previous literature and compare our results with it. First we examine experiments on ambiguity in games, follwing which we consider experiments related to the Ellsberg urn.

\subsection{Papers on Games}

One previous experimental study of the effect of ambiguity in games is Di Mauro and Castro (2011). They test a result from Eichberger and Kelsey (2002), concerning the effect of ambiguity on the voluntary provision of public goods. Eichberger and Kelsey (2002) show that when the

\footnotetext{
${ }^{22}$ We do not consider $x=60$, where $R$ is a dominated strategy.
} 
production function for public goods is concave, ambiguity-aversion causes public good provision to be above the Nash equilibrium level. More generally they show that the deviation from the Nash equilibrium depends on the nature of strategic interactions taking place, i.e., on the basis of whether the game being played was one of strategic substitutes or complements.

Di Mauro and Castro (2011) test this hypothesis in an experiment on voluntary provision of public goods, which is a game of strategic substitutes. They show that ambiguity rather than altruism causes an increase in contribution towards the public good. In order to negate the chance that altruism, or a feeling of reciprocation prompted the subjects' actions, they were informed that their opponent would be a virtual agent and the opponent's play was simulated by a computer. Subjects played in two scenarios, one with risk, the other with ambiguity. Contributions were significantly higher when the situation was one of ambiguity. These results are similar to our findings and showed that there was indeed evidence that ambiguity significantly affects the decisions made by individuals, in a manner that depends directly on the strategic nature of the game in consideration.

Another paper that tests whether ambiguity affects behaviour in games is Eichberger, Kelsey, and Schipper (2008). They studied games in which subjects faced either a granny, who was described as being ignorant of economic strategy, a game theorist, who was described as a successful professor of economics, or another student as an opponent. It was conjectured that subjects would view the granny as a more ambiguous opponent than the game theorist. This was confirmed by the data and ambiguity affected decision choices in the predicted way. In our paper, even though subjects are paired against one another (and not a granny), we find subjects display similar ambiguity averse behaviour.

Colman and Pulford (2007) explain the concept of ambiguity aversion as a state that arises as a result of a pessimistic response to uncertainty. This is mainly driven by a loss of decision confidence. They argue that people tend to become anxious and less confident while making decisions in the presence of ambiguity. They found that individual responses differed between ambiguous and risky versions of the game being studied. Players did not respond to ambiguity by simply equating it to riskiness, but showed a marked preference to avoid ambiguity whenever 
the option of doing so was provided to them. This is consistent with our findings that when an ambiguity-safe option is made available to subjects, they show a marked preference for it.

\subsection{Papers on Ellsberg Urns}

One of the earliest Ellsberg experiments was conducted by Becker and Brownson (1964). Ambiguity was implemented as the number of red balls in an urn. When given the choice between an ambiguous urn and an unambiguous one, subjects chose the unambiguous urn and were willing to pay an ambiguity premium of about $60 \%$ to avoid ambiguity. Another Ellsberg experiment conducted by Yates and Zukowski (1976), found subjects to be willing to pay a premium of $20 \%$ of the expected value of a bet, in order to bet on a known urn instead of a uniform one. In our experiments, we found that most subjects were unwilling to bear even the smallest penalty in order to avoid ambiguity and choose Red.

Bernasconi and Loomes (1992), study a two-stage lottery version of the three-colour Ellsberg problem, where drawing a red ball $(p(R)=1 / 3)$ was the unambiguous event, while drawing blue and yellow balls were ambiguous separately, but unambiguous together, $(p(B \cup Y)=2 / 3)$. Ambiguity aversion was displayed by half the subjects who chose to bet on red for $£ 10$. In addition, $90 \%$ of subjects who chose a $£ 10$ bet on red, refused to switch to a $£ 12$ bet on an ambiguous colour - thereby implying an ambiguity premium of about $20 \%$. In our experiment, we framed the ambiguity premium as a penalty on Red (the unambiguous ball) and found that subjects preferred instead to bet on Blue/Yellow i.e., the ambiguous balls.

\section{Conclusions}

The Nash equilibrium prediction that $R$ cannot be chosen for $150<x<300$, was not observed in our experiments. The ambiguity-safe option $R$, which is selected by EUA, was the choice of a majority of subjects when $120<x<260$. There was also a significant minority of subjects choosing $R$ when $x=60$. Thus, we find evidence which is consistent with EUA predictions.

A possible means of testing whether it was indeed ambiguity-aversion prompting the choice 
of $R$, would be to run our game in a corresponding "risky" set-up. Analogous to Bohnet, Greig, Herrmann, and Zeckhauser (2008), there could be a condition where the Column Player is told that he is matched against a Computer playing Row. ${ }^{23}$ Moreover, he is told that the Computer is playing a mixed strategy, defined by the empirical frequency of the human Row Players for any given value of $x$. If the Column Player does not choose $R$ when $x>100$ in the computerised-treatments, it would suggest that his choices in our original-setup experiment are driven by something other than pure risk-aversion, strengthening our argument that ambiguityaversion is at work. However, this is beyond the scope of the present paper and may be an avenue for future research.

One surprising feature of our results was that the links between choices in the single person decision and those in the games was not strong. Subjects appeared to perceive a greater level of ambiguity in a two-person coordination game, than a single person decision problem. More generally our results suggest that perceptions of ambiguity and even attitudes to ambiguity depend on context. Hence it may not be possible to measure ambiguity-attitude in one context and use it to predict behaviour in another.

It is interesting to note that subjects find more ambiguity regarding real events as opposed to actual or simulated Ellsberg urns. It was found that when Ellsberg-type problems were put to students in a class-environment, a large proportion of PhD-level students were ambiguity-neutral, while a large proportion of MBA-level students displayed ambiguity-seeking behaviour. ${ }^{24}$ However, when asked whether they preferred a payoff of $\$ 100$ if the US President elected in 2016 was a Democrat (or not a Democrat) or if a fair coin came up heads when tossed on the day of the election, a large proportion of the students preferred betting on the coin.

One of the reasons put forth to explain this divergence in behaviour is that it is easy to be Bayesian in an Ellsberg experiment or that the phrasing of the Ellsberg problem might lead to it being treated as a gamble. However, when asked to make a decision regarding a realistic scenario such as predicting the next President of the US, the students have no "natural" prior.

\footnotetext{
${ }^{23}$ We would like to thank our anonymous referee for this suggestion.

${ }^{24}$ These observations are as recorded by Itzhak Gilboa, in a discussion on observed ambiguity in Ellsberg experiments.
} 
A realistic scenario then is better at revealing ambiguity aversion on the part of the subject.

Parallels can be drawn between this discussion and the data we observe from our experiment, whereby subjects clearly display ambiguity-averse behaviour when put in the scenario of the coordination game while they fail to do so in the Ellsberg urn rounds. Subjects might be treating the Ellsberg urn rounds as a gamble, where they readily take a chance. However, when faced with the task of coordinating with another participant in the environment of a one-shot game with no previous learning, the subjects have no natural prior on the basis of which to make their decisions. The Column Player thus selects the strategy that gives a definite payoff of $x$ irrespective of the Row Player's decision.

One can note that our results support the Dow and Werlang (1994) model of equilibrium under ambiguity, where in the presence of ambiguity players choose their safe strategy, rather than the model of Lo (1996). Lo's equilibrium predictions coincide with those of Nash equilibrium for games with only pure equilibria. Thus for many of our game experiments Lo's predictions coincide with Nash equilibrium. Hence for these experiments EUA appears to predict the implications of ambiguity better.

\section{A Appendix}

Proof of Proposition 3.1: $\quad$ Part (1) $\quad 0 \leqslant x<75$ : By inspection $(T, M)$ and $(B, L)$ are pure strategy Nash equilibria. Let the probability of Player 1 choosing $T$ (resp. $B$ ) be $q$ (resp. $(1-q))$, and the probability of Player 2 choosing $L(\operatorname{resp} . M)$ be $p(\operatorname{resp} .(1-p))$. For $x$ in this range, $R$ is dominated by $\frac{1}{4} \cdot L+\frac{3}{4} \cdot M$, which yields an expected pay-off of 75 . Thus $R$ cannot be played in Nash equilibrium. There are 3 equilibria: $(T, M),(B, L)$ and $\left(\frac{3}{4} \cdot T+\frac{1}{4} \cdot B, \frac{1}{4} \cdot L+\frac{3}{4} \cdot M\right)$.

Part (2) $75<x \leqslant 100$ : For $x$ in this range, $(T, M)$ and $(B, L)$ remain pure strategy Nash equilibria. Player 2 is indifferent between $M$ and $R$ when: $100 q=x$ or $q=x / 100$. Player 1 is indifferent between $T$ and $B$ when: $300 p+50(1-p)=55(1-p)$, or $p=1 / 61$. There are 3 equilibria: $(T, M),(B, L)$ and $\left(\frac{x}{100} \cdot T+\frac{100-x}{100} \cdot B, \frac{1}{61} \cdot M+\frac{60}{61} \cdot R\right) .{ }^{25}$

\footnotetext{
${ }^{25}$ There are no equilibria where Player 2 mixes between $L$ and $R$. Such an equilibrium would require Player
} 
Part (3) $\quad 100<x<300$ : For this range, $M$ is dominated for Player 2 by $R$. Once $M$ is eliminated, Player 1 will never play $T$, which is now a dominated strategy. He thus plays $B$. The best response for Player 2 is to play $L$. In this case there is a unique Nash equilibrium: $(B, L)$, which is also an iterated dominance equilibrium.

Proof of Proposition 3.2: $\quad$ Part 1. $\quad 0 \leqslant x \leqslant\left(1-\delta_{i}\right) 75$ : In this range there are two EUA in pure strategies and one in mixed strategies. In the pure equilibria, the supports of the equilibrium beliefs are given by $(T, M)$ and $(B, L)$. Consider the first of these. Define a capacity, $\nu_{1}$, by $\nu_{1}=\left(1-\delta_{1}\right) \pi_{M}(A)$, where $\pi_{M}$ is the additive probability on $S_{2}$ defined by $\pi_{M}(A)=1$ if $M \in A, \pi_{M}(A)=0$ otherwise. Similarly define Player 2's beliefs $\nu_{2}$ by $\nu_{2}=\left(1-\delta_{2}\right) \pi_{T}(A)$. By definition $\operatorname{supp} \nu_{1}=M$ and $\operatorname{supp} \nu_{2}=T$. Denote this equilibrium by $\langle T, M\rangle$. By similar reasoning we may show that there exists a pure equilibrium where $\operatorname{supp} \nu_{1}=L$ and $\operatorname{supp} \nu_{2}=B$, which we denote by $\langle B, L\rangle$.

Now consider the mixed equilibria. Denote the equilibrium beliefs of Players 1 and 2 respectively by $\tilde{\nu}_{1}=\left(1-\tilde{\delta}_{1}\right) \tilde{\pi}_{1}$ and $\tilde{\nu}_{2}=\left(1-\tilde{\delta}_{2}\right) \tilde{\pi}_{2}$. Player 2's Choquet expected pay-offs are given by, $V_{2}(L)=300\left(1-\tilde{\delta}_{2}\right) \tilde{\pi}_{2}(B), V_{2}(M)=100\left(1-\tilde{\delta}_{2}\right) \tilde{\pi}_{2}(T)$ and $V_{2}(R)=x$. If $V_{2}(L)<x \leqslant\left(1-\tilde{\delta}_{2}\right) 75$ then $\tilde{\pi}_{2}(B)<\frac{1}{4}$, which implies $\tilde{\pi}_{2}(T)>\frac{3}{4}$. Hence $V_{2}(M)=$ $100\left(1-\tilde{\delta}_{2}\right) \tilde{\pi}_{2}(T)>\left(1-\tilde{\delta}_{2}\right) 75 \geqslant x$. Thus $R$ cannot be a best response for Player 2 , hence $\tilde{\pi}_{1}(R)=0$. Consequently in any mixed equilibrium 2's strategies are $L$ and $M$.

In a mixed equilibrium Player 2 must be indifferent between $L$ and $M$, hence,

$$
\begin{aligned}
V_{2}(L) & =V_{2}(M) \Leftrightarrow 300\left(1-\tilde{\delta}_{2}\right) \tilde{\pi}_{2}(B)=100\left(1-\tilde{\delta}_{2}\right) \tilde{\pi}_{2}(T) \\
& \Leftrightarrow \quad \tilde{\pi}_{2}(T)=\frac{3}{4} .
\end{aligned}
$$

In this equilibrium $V_{2}(L)=V_{2}(M)=75\left(1-\tilde{\delta}_{2}\right)$. Similarly we may show that for Player 1 to be indifferent between $T$ and $B$, we must have $\tilde{\pi}_{1}(L)=\frac{1}{4}$ and $\tilde{\pi}_{1}(M)=\frac{3}{4}$.

Thus in the mixed equilibrium $\tilde{\nu}_{1}=\left(1-\tilde{\delta}_{1}\right) \tilde{\pi}_{1}$ with $\tilde{\pi}_{1}(L)=\frac{1}{4}$ and $\tilde{\pi}_{1}(M)=\frac{3}{4}$ and

2 to be indifferent between $L$ and $R$ when: $300(1-q)=x$ or $q=\frac{300-x}{300}$. Player 1 is indifferent between $T$ and $B$ when: $100 p+55(1-p)=50(1-p)$. However there is no solution to this equation with a positive value of $p$, $\left(p=-\frac{5}{95}\right.$ is a solution).. 
$\operatorname{supp} \tilde{\nu}_{1}=\{L, M\}$ while $\tilde{\nu}_{2}=\left(1-\tilde{\delta}_{2}\right) \tilde{\pi}_{2}$ with $\tilde{\pi}_{2}(T)=\frac{3}{4}$ and $\tilde{\pi}_{2}(B)=\frac{1}{4}$, with support $\{T, B\}$. In this equilibrium $V_{2}(L)=V_{2}(M)=75\left(1-\tilde{\delta}_{2}\right)$. We shall denote this equilibrium by $\left\langle\frac{3}{4} \circ T+\frac{1}{4} \circ B, \frac{1}{4} \circ L+\frac{3}{4} \circ M\right\rangle$.

Part 2. $\quad\left(1-\delta_{i}\right) 75<x<\left(1-\delta_{i}\right) 100: \quad$ In this range, there are two EUA in pure strategies: $(T, M)$ and $(B, L)$. The reasoning is similar to that used in Part 1 above.

In addition, there is a mixed strategy equilibrium. Denote the equilibrium beliefs of Players 1 and 2 respectively by $\tilde{\nu}_{1}=\left(1-\tilde{\delta}_{1}\right) \tilde{\pi}_{1}$ and $\tilde{\nu}_{2}=\left(1-\tilde{\delta}_{2}\right) \tilde{\pi}_{2}$. Player 2's Choquet expected payoffs are given by, $V_{2}(L)=300\left(1-\tilde{\delta}_{2}\right) \tilde{\pi}_{2}(B), V_{2}(M)=100\left(1-\tilde{\delta}_{2}\right) \tilde{\pi}_{2}(T)$ and $V_{2}(R)=x$. Thus $L$ cannot be a best response for Player 2 , hence $\tilde{\pi}_{1}(L)=0 .{ }^{26}$ Consequently in any mixed equilibrium 2's strategies are $M$ and $R$.

Player 2 is indifferent between $M$ and $R$ when:

$$
\begin{aligned}
V_{2}(M) & =V_{2}(R) \Leftrightarrow 100\left(1-\tilde{\delta}_{2}\right) \tilde{\pi}_{2}(T)=x \\
& \Leftrightarrow \quad \tilde{\pi}_{2}(T)=\frac{x}{\left(1-\tilde{\delta}_{2}\right) 100}
\end{aligned}
$$

Similarly, Player 1's Choquet expected payoff is given by: $V_{1}(T)=300\left(1-\tilde{\delta}_{1}\right) \tilde{\pi}_{1}(M)+$ $50\left(1-\tilde{\delta}_{1}\right) \tilde{\pi}_{1}(R)$ and $V_{1}(B)=55\left(1-\tilde{\delta}_{1}\right) \tilde{\pi}_{1}(R)$. Player 1 is indifferent between $T$ and $B$ when:

$$
\begin{aligned}
V_{1}(T) & =V_{1}(B) \\
& \Leftrightarrow 300\left(1-\tilde{\delta}_{1}\right) \tilde{\pi}_{1}(M)+50\left(1-\tilde{\delta}_{1}\right) \tilde{\pi}_{1}(R)=55\left(1-\tilde{\delta}_{1}\right) \tilde{\pi}_{1}(R) \\
& \Leftrightarrow \tilde{\pi}_{1}(M)=\frac{1}{61} .
\end{aligned}
$$

\footnotetext{
${ }^{26}$ Consider what would happen if Player 2 mixes between $L$ and $R$. For Player 2 to be indifferent between $L$ and $R$ :

$$
V_{2}(L)=V_{2}(R) \Leftrightarrow 300\left(1-\tilde{\delta}_{2}\right) \tilde{\pi}_{2}(B)=x \Leftrightarrow \tilde{\pi}_{2}(B)=\frac{x}{300\left(1-\tilde{\delta}_{2}\right)} .
$$
}

Player 1 is then indifferent between playing $T$ and $B$ when,

$$
\begin{aligned}
V_{1}(T) & =V_{1}(B) \Leftrightarrow 50\left(1-\tilde{\delta}_{1}\right) \tilde{\pi}_{1}(R)=100\left(1-\tilde{\delta}_{1}\right) \tilde{\pi}_{1}(L)+55\left(1-\tilde{\delta}_{1}\right) \tilde{\pi}_{1}(R) \\
& \Leftrightarrow 100 \tilde{\pi}_{1}(L)=-5\left(1-\tilde{\pi}_{1}(L) \Leftrightarrow \tilde{\pi}_{1}(L)=-\frac{5}{95} .\right.
\end{aligned}
$$

It is impossible for a belief to be negative, hence there can be no such equilibria. 
Thus in the mixed equilibrium $\tilde{\nu}_{1}=\left(1-\tilde{\delta}_{1}\right) \tilde{\pi}_{1}$, with $\tilde{\pi}_{1}(M)=\frac{1}{61}$ and $\tilde{\pi}_{1}(R)=\frac{60}{61}$ and $\operatorname{supp} \tilde{\nu}_{1}=\{M, R\}$, while $\tilde{\nu}_{2}=\left(1-\tilde{\delta}_{2}\right) \tilde{\pi}^{2}$ with $\tilde{\pi}_{2}(T)=\frac{x}{\left(1-\tilde{\delta}_{2}\right) 100}$ and $\tilde{\pi}_{2}(B)=\frac{\left(1-\tilde{\delta}_{2}\right) 100-x}{\left(1-\tilde{\delta}_{2}\right) 100}$, with support $\{T, B\}$. In this equilibrium $V_{2}(M)=V_{2}(R)=x$. The mixed strategy equilibrium is $\left\langle\frac{x}{\left(1-\tilde{\delta}_{2}\right) 100} \circ T+\frac{\left(1-\tilde{\delta}_{2}\right) 100-x}{\left(1-\tilde{\delta}_{2}\right) 100} \circ B, \frac{1}{61} \circ M+\frac{60}{61} \circ R\right\rangle$.

Part 3. $\quad\left(1-\delta_{i}\right) 100<x<\left(1-\delta_{i}\right) 300$ : Denote the equilibrium beliefs of Players 1 and 2 respectively by $\tilde{\nu}_{1}=\left(1-\tilde{\delta}_{1}\right) \tilde{\pi}_{1}$ and $\tilde{\nu}_{2}=\left(1-\tilde{\delta}_{2}\right) \tilde{\pi}_{2}$. Player 2's Choquet expected pay-offs are given by, $V_{2}(L)=300\left(1-\tilde{\delta}_{2}\right) \tilde{\pi}_{2}(B), V_{2}(M)=100\left(1-\tilde{\delta}_{2}\right) \tilde{\pi}_{2}(T)$ and $V_{2}(R)=x$.

For $x$ in this range, $V_{2}(R)>V_{2}(M)$ for any beliefs of Player 2, hence $\tilde{\pi}_{1}(M)=0$. Player 1's Choquet expected pay-offs are given by, $V_{1}(T)=50\left(1-\tilde{\delta}_{1}\right) \tilde{\pi}_{1}(R)$ and $V_{1}(B)=100\left(1-\tilde{\delta}_{1}\right) \tilde{\pi}_{1}(L)+$ $55\left(1-\tilde{\delta}_{1}\right) \tilde{\pi}_{1}(R)$. Strategy $B$ yields a higher Choquet expected payoff than $T$ for any beliefs of Player 1, with support contained in $\{L, R\}$. For Player $2, L$ is the best response to $B$. In this case there is a unique EUA: $\langle B, L\rangle$.

Part 4. $\quad x>\left(1-\delta_{i}\right) 300$ : Denote the equilibrium beliefs of Players 1 and 2 respectively by $\tilde{\nu}_{1}=\left(1-\tilde{\delta}_{1}\right) \tilde{\pi}_{1}$ and $\tilde{\nu}_{2}=\left(1-\tilde{\delta}_{2}\right) \tilde{\pi}_{2}$. Player 2's Choquet expected pay-offs are given by, $V_{2}(L)=300\left(1-\tilde{\delta}_{2}\right) \tilde{\pi}_{2}(B), V_{2}(M)=100\left(1-\tilde{\delta}_{2}\right) \tilde{\pi}_{2}(T)$ and $V_{2}(R)=x$, where $x>$ $\left(1-\tilde{\delta}_{2}\right) 300$.

For $x$ in this range, $R$ strictly dominates both $L$ and $M$ for any beliefs of Player 2, hence $\tilde{\pi}_{1}(L)=\tilde{\pi}_{1}(M)=0$. Player 1's best response is to play $B$, with $\operatorname{supp} \nu_{1}=R$. There is a unique EUA: $\langle B, R\rangle$.

\section{References}

Becker, S., and F. Brownson (1964): "What Price Ambiguity? Or the Role of Ambiguity in Decision Making," Journal of Political Economy, 72, 62-73.

Bernasconi, M., and G. Loomes (1992): "Failures of the Reduction Principle in an Ellsberg Type Problem," Theory and Decision, 32, 77-100.

Bohnet, I., F. Greig, B. Herrmann, and R. Zeckhauser (2008): "Betrayal Aversion: Evidence from Brazil, China, Oman, Switzerland, Turkey, and the United States," American 
Economic Review, 98(1), $294-310$.

Camerer, C., T.-H. Ho, and J.-K. Chong (2004): "A Cognitive Hierarchy Model of Games," Quarterly Journal of Economics, 119 (3), 861-898.

Camerer, C. F., and M. Weber (1992): "Recent Developments in Modelling Preferences: Uncertainty and Ambiguity," Journal of Risk and Uncertainty, 5, 325-370.

Chateauneuf, A., J. Eichberger, and S. Grant (2007): "Choice under Uncertainty with the Best and Worst in Mind: NEO-Additive Capacities," Journal of Economic Theory, 137, $538-567$.

Colman, A., and B. Pulford (2007): "Ambiguous Games: Evidence for Strategic Ambiguity Aversion," Quarterly Journal of Experimental Psychology, 60, 1083-1100.

Di Mauro, C., and M. F. Castro (2011): "Kindness Confusion or ... Ambiguity," Experimental Economics, 14, 611-633.

Dow, J., And S. R. C. Werlang (1994): "Nash Equilibrium under Uncertainty: Breaking Down Backward Induction," Journal of Economic Theory, 64, 305-324.

Eichberger, J., And D. Kelsey (2000): "Non-Additive Beliefs and Strategic Equilibria," Games and Economic Behavior, 30, 183-215.

(2002): "Strategic Complements, Substitutes and Ambiguity: The Implications for Public Goods," Journal of Economic Theory, 106, 436-466.

(2014): “Optimism and Pessimism in Games," International Economic Review, 55, $483-505$.

Eichberger, J., D. Kelsey, and B. Schipper (2008): "Granny Versus Game Theorist: Ambiguity in Experimental Games," Theory and Decision, 64, 333-362.

(2009): "Ambiguity and Social Interaction," Oxford Economic Papers, 61, 355-379. 
GilboA, I., And D. Schmeidler (1989): "Maxmin Expected Utility with a Non-Unique Prior," Journal of Mathematical Economics, 18, 141-153.

Ivanov, A. (2011): "Attitudes to Ambiguity in One-Shot Normal Form Games: An Experimental Study," Games and Economic Behavior, 71, 366-394.

Kelsey, D., And S. Le Roux (2013): "Dragon Slaying and Dyke Building - How Does Ambiguity Affect Individual Behaviour?,"SSRN: http://ssrn.com/abstract=2320519.

Kilka, M., And M. Weber (2001): "What Determines the Shape of the Probability Weighting Function under Uncertainty?," Management Science, 47, 1712-1726.

Klibanoff, P. (1993): "Uncertainty, Decision and Normal Form Games," working paper, Northwestern University.

Lo, K. C. (1996): "Equilibrium in Beliefs under Uncertainty," Journal of Economic Theory, 71, 443-484.

Savage, L. J. (1954): Foundations of Statistics. Wiley, New York.

Schmeidler, D. (1989): "Subjective Probability and Expected Utility without Additivity," Econometrica, 57, 571-587.

YAtes, J. F., AND L. ZukOWski (1976): "Characterisation of Ambiguity in Decision Making," Behavioral Science, 21, 19-25. 